\title{
The physical therapist's role in physical activity promotion
}

\author{
E Verhagen, ${ }^{1} \mathrm{~L}$ Engbers ${ }^{2}$
}

${ }^{1}$ Department of Public and Occupational Health, EMGOInstitute, VU University Medical Centre, Amsterdam, The Netherlands; ${ }^{2}$ TNO Quality of Life, Leiden, The Netherlands

Correspondence to:

Dr E Verhagen, Department of Public and Occupational Health, EMGO-Institute, VU University Medical Centre, Amsterdam, The Netherlands; e.verhagen@ vumc.nl

Accepted 18 September 2008 Published Online First 6 October 2008

\section{ABSTRACT}

Clinicians are increasingly confronted with the diseases of physical inactivity. Paradoxically, a promising strategy to motivate sedentary individuals to become more active is the opportunity to encourage physical activity related behavioural change when individuals encounter health professionals. Although health care professionals trained in exercise prescription are recognised as having the potential to play an important role in promoting regular physical activity, their role has been minimal in multidisciplinary approaches to date. Even so, the role of physical therapists has gone largely unrecognised. Nevertheless, because of their training and experience, physical therapists are ideally placed to promote the health and well being of individuals and the general public through physical activity and exercise prescription. Therefore, physical therapists, general practitioners and other caregivers alike should become aware of the physical therapist's potential in promoting safe and healthy physical activity.

Worldwide physical inactivity and the associated health problems are a current and still growing threat to public health. For example, $46.5 \%$ and $11.3 \%$ of the Dutch population suffers from overweight or obesity, respectively. ${ }^{1}$ The level of physical activity that is deemed sufficient is clearly described and well communicated to the Dutch population through various public channels. Nevertheless, only $56 \%$ of the Dutch population achieves this recommended level of physical activity. ${ }^{2}$ Therefore, clinicians are increasingly confronted with the diseases of physical inactivity.

Paradoxically, a promising strategy to persuade sedentary individuals to become more active is the opportunity to encourage physical activity related behavioural change when individuals encounter health professionals. ${ }^{3}$ As the majority of the adult population visits a general practitioner (GP) each year, it is not surprising that most research has focused on the role of the GP in promoting physical activity. ${ }^{4}$ Counselling interventions by GPs are effective in increasing physical activity. However, these encouraging changes have generally not been long-lasting. ${ }^{5}$ For example, an Australian study showed very promising results in terms of increased awareness among professionals in their role as physical activity promoters. ${ }^{6}$ In the "10,000 Steps Rockhampton" community intervention, different strategies targeting GPs were evaluated. A matched community was used as a comparison. High participation and intervention uptake among GPs and a significant increase in the number of community residents counselled on physical activity were clear indicators of the success of this project.

Is there room for a multidisciplinary approach in which numerous health professionals play a role in the promotion of physical activity? Although health care professionals trained in exercise prescription are recognised as having the potential to play an important role in promoting regular physical activity, ${ }^{7}$ their role has been minimal in multidisciplinary approaches to date. The purpose of this article is to focus on the role that the physical therapist may play in promoting physical activity. Despite the evidence that physical therapists can provide effective physical activity promotion, ${ }^{8}$ this role has gone largely unrecognised by members of the public, other healthcare professionals and, we believe, by physical therapists themselves.

\section{PHYSICAL THERAPY, HEALTH AND THE MOVING BODY}

The overall and universal aim of physical therapy is to identify and maximise human movement potential within the spheres of promotion, prevention, treatment and rehabilitation. This prominently includes providing services in circumstances where movement and function are threatened by, for example. ageing, injury, disease or environmental factors. Furthermore, physical therapy always involves the interaction between therapist, patient, other health professionals, families, caregivers and communities in a process where movement potential is assessed and goals are agreed upon. ${ }^{9}$

According to the position statement of the World Confederation for Physical Therapy, physical therapists may be concerned in a variety of settings with any of the following purposes?

- promoting the health and well being of individuals and the general public/society, emphasising the importance of physical activity and exercise

- preventing impairments, activity limitations, participatory restrictions and disabilities in individuals at risk of altered movement behaviours due to health or medically related factors, socioeconomic stressors, environmental factors and lifestyle factors

- providing interventions/treatment to restore integrity of body systems essential to movement, maximise function and recuperation, minimise incapacity, and enhance the quality of life, independent living and workability in individuals and groups of individuals with altered movement behaviours resulting from 
impairments, activity limitations, participatory restrictions and disabilities

- modifying environmental, home and work access and barriers to ensure full participation in one's normal and expected societal roles.

Thus, it is clear that the physical therapist is a health care professional specialising in human movement and its relationship with health. Additionally, physical therapists are trained to prescribe safe physical activity even among the unwell and frail. This makes physical therapists ideal for promoting safe and healthy physical activities.

\section{PHYSICAL THERAPY AND THE PROMOTION OF PHYSICAL ACTIVITY}

Although physical therapists should be committed to promoting physical activity and exercise, ${ }^{9}$ in practice their services generally focus on restoration and maintenance of optimal function and quality of life in individuals with loss and disorders of movement. This secondary and tertiary prevention for individuals with impaired movement due to a wide variety of causes may be driven to a large extent by payment structures. Most health insurers do not pay for "prevention" or "exercise prescription". This significantly narrows down the physical therapist's scope to promote physical activity for general health reasons.

Because of this "disease based" model, most patients attend the physical therapist for specific movement related problems, limiting the physical therapist to the role of caregiver. In the current paradigm, in many consultations it is unlikely that physical activity promotion will be initiated. For example, consider a patient with tennis elbow presenting to a physical therapist. The injury may have been exacerbated by the physical demands of his job; this man also has a body mass index of $32 \mathrm{~kg} / \mathrm{m}^{2}$ and is inactive. If we assume that this medical problem is not directly due to the obesity, then it raises the issue of whether the physical therapist should promote physical activity for this man's overweight. Informing the patient about the importance of physical activity for general health and on the risks of being overweight is clearly within the physical therapist's skills and scope. However, actually adjusting the treatment plan to address this man's weight problem (for example, through counselling, or by providing general exercise protocols) might not be appropriate or feasible for the reasons that follow.

First of all, the time available for the physical therapist to treat a medical problem is restricted, because most health care insurances only cover a certain amount of treatments for specific disorders. Secondly, although physical therapists are autonomous in many aspects of their work, in most countries it is the GP who, to a large extent, determines the treatment protocol, and promotion of physical activity might not fall within the "best practice" treatment prescribed by the GP. Moreover, for a treatment to be successful, in this case physical activity promotion, it needs to fall within the scope of the patient's expectations, wishes, motivation, and goals. Therefore, in this specific example, the physical therapist will not quickly decide to start physical activity promotion.

It would be different if the same man was visiting the physical therapist because of osteoarthritis. In this case, it is well known that moderate physical activity and subsequent weight loss improves symptoms, as well as psychosocial and functional status. ${ }^{10}$ It would be almost negligent if the physical therapist did not include physical activity promotion in the treatment plan for such a patient. These contrasting examples clearly illustrate the problems physical therapists face when trying to promote physical activity. Physical therapists are visited by patients with specific movement related medical problems, and not by symptom-free "at risk" individuals who need to be more active. Therefore, promotion of physical activity by a physical therapist can, in current practice, only be undertaken from the perspective of secondary and tertiary prevention.

\section{HOW TO UNLOCK THE PHYSICAL THERAPIST'S POTENTIAL}

Current insights into physical activity promotion emphasise that success requires a multidisciplinary approach. Due to the prevalence of obesity and subsequent inactivity related health problems, all health professionals must promote physical activity. Should it be mandatory for every health professional to be trained in (basic) exercise prescription, ${ }^{4}$ or is it sufficient that certain health professionals, such as physical therapists, receive particularly extensive training in physical activity and exercise prescription?

There is a case for physical activity counsellors to be integrated into general practice where they would provide ongoing, possibly intensive, individual physical activity guidance. ${ }^{11} 12$ These physical activity counsellors need to be trained in the field of exercise science, and more importantly need to be knowledgeable about local physical activity resources. ${ }^{13}$

Physical therapists also fit this profile as they already train patients (for example, group exercise sessions), transfer knowledge and prescribe physical activity on a regular basis to their current patients. When GPs refer patients in need of physical activity guidance to a local physical therapist, this individual is perfectly capable of taking up the role of physical activity counsellor for the wider general public. This has been found to be the case in, to our knowledge, the only physical therapy specific study on physical activity promotion, from which it was concluded that physical therapists are a capable group ready to engage in physical activity promotion. ${ }^{8}$

It remains debatable whether this referral should go for all patients in need of physical activity counselling, or if the GP should only refer the more frail and sicker patients to a physical therapist, while the "healthier" patients are directed to a physical activity counsellor. Nonetheless, it is unequivocal that the role of the physical therapist in physical activity promotion should be further exploited and investigated before effort and finances are put into the creation of "new" physical activity oriented health professionals.

\section{CONCLUSIONS}

- In current practice, physical therapists generally provide secondary and tertiary prevention for individuals who have impaired movement due to a wide variety of causes that may or may not be related to physical inactivity. This severely limits the scope of physical therapists and their opportunity to promote physical activity for general health.

- Because of their training and experience, physical therapists are ideally placed to promote the health and well being of individuals and the general public through physical activity and exercise prescription.

- Physical therapists, GPs and other caregivers alike should become aware of the potential of physical therapists in promoting safe and healthy physical activity.

Competing interests: None. 


\section{REFERENCES}

1. CBS Statline. Gerapporteerde gezondheid en leefstijl, www.cbs.nl 14-05-2007.

2. Trendrapport Bewegen en Gezondheid 2004/2005. TNO Kwaliteit van Leven. De Bink Leiden, 2007.

3. Smith BJ. Promotion of physical activity in primary health care: an update of the evidence of interventions. J Sci Med Sport 2004;7(supl 1):67-73.

4. Brown WJ. Individual or population approaches to the promotion of physical activity...is that the question?J Sci Med Sport 2006;9:35-7

5. Riddoch C, Puig-Ribera A, Cooper A. Effectiveness of physical activity promotion schemes in primary care: a review. London: Health Education Authority, 1998.

6. Brown WJ, Mummery K, Eakin E, et al. 10,000 Steps Rockhampton: evaluation of a whole community approach to improving population levels of physical activity. J Physical Activity Health 2006;3:1-14.

7. Owen N. Strategic initiatives to promote participation in physical activity. Health Promotion International 1996;11:213-9.
8. Sheedy J, Smith B, Bauman A, et al. A controlled trial of behavioural education to promote exercise among physiotherapy outpatients. Aust $J$ Physiother 2000;46:281-9.

9. World Confederation of Physical Therapy. Position Statement, 2007. http://www.wcpt.org/common/docs/policies/Description\%20of\%20Physical\%20 Therapy\%20-\%20Sep\%2007\%20Rev\%202.pdf

10. Minor M. Physical activity and the management of arthritis. Ann Behav Med 1991;13:117-24.

11. Tulloch $\mathbf{H}$, Fortier $M$, Hogg W. Physical activity counseling in primary care: who has and who should counsel? Patient Educ Couns 2006;64(1-3):6-20.

12. Sorensen JB, Skovgaard T, Puggaard L. Exercise on prescription in general practice: a systematic review. Prim Health Care 2006;24:69-74.

13. Fortier $\mathbf{M}$, Tulloch $\mathrm{H}$, Hogg W. A good fit. Integrating physical activity counselors into family practice. Can Fam Physician 2006;52:942-4. 


\title{
BJSM
}

\section{The physical therapist's role in physical activity promotion}

\author{
E Verhagen and L Engbers
}

Br J Sports Med 2009 43: 99-101 originally published online October 6, 2008

doi: $10.1136 /$ bjsm.2008.053801

Updated information and services can be found at:

http://bjsm.bmj.com/content/43/2/99.full.html

These include:

References This article cites 9 articles, 2 of which can be accessed free at: http://bjsm.bmj.com/content/43/2/99.full.html\#ref-list-1

Article cited in:

http://bjsm.bmj.com/content/43/2/99.full.html\#related-urls

Email alerting Receive free email alerts when new articles cite this article. Sign up in service the box at the top right corner of the online article.

Notes

To request permissions go to:

http://group.bmj.com/group/rights-licensing/permissions

To order reprints go to:

http://journals.bmj.com/cgi/reprintform

To subscribe to BMJ go to:

http://group.bmj.com/subscribe/ 